\title{
Phosphine in Venus' atmosphere: Detection attempts and upper limits above the cloud top assessed from the SOIR/VEx spectra
}

\author{
L. Trompet ${ }^{1}$, S. Robert ${ }^{1,2}$, A. Mahieux ${ }^{1,3}$, F. Schmidt ${ }^{4}$, J. Erwin ${ }^{1}$, and A. C. Vandaele ${ }^{1}$ \\ 1 Planetary Aeronomy, Royal Belgian Institute for Space Aeronomy, Brussels, Belgium \\ e-mail: loic.trompet@aeronomie.be \\ 2 Institute of Condensed Matter and Nanosciences, Université catholique de Louvain, Chemin du Cyclotron 2, \\ 1348 Louvain-la-Neuve, Belgium \\ 3 The University of Texas at Austin, Austin, TX, USA \\ 4 Université Paris-Saclay, CNRS, GEOPS, 91405 Orsay, France
}

Received 17 November 2020 / Accepted 10 December 2020

\begin{abstract}
Context. Recent detection of phosphine $\left(\mathrm{PH}_{3}\right)$ was reported from James Clerk Maxwell Telescope and Atacama Large Millimetre/submillimetre Array observations. The presence of $\mathrm{PH}_{3}$ on Venus cannot be easily explained in the Venus atmosphere and a biogenic source located at or within the clouds was proposed.

Aims. We aim to verify if the infrared spectral signature of $\mathrm{PH}_{3}$ is present in the spectra of Solar Occultation at Infrared (SOIR). If it is not present, we then seek to derive the upper limits of $\mathrm{PH}_{3}$ from SOIR spectra.

Methods. We analyzed the SOIR spectra containing absorption lines of $\mathrm{PH}_{3}$. We searched for the presence of $\mathrm{PH}_{3}$ lines. If we did not find any conclusive $\mathrm{PH}_{3}$ spectral signatures, we computed the upper limits of $\mathrm{PH}_{3}$.

Results. We report no detection of $\mathrm{PH}_{3}$. Upper limits could be determined for all of the observations, providing strong constraints on the vertical profile of $\mathrm{PH}_{3}$ above the clouds.

Conclusions. The SOIR $\mathrm{PH}_{3}$ upper limits are almost two orders of magnitude below the announced detection of $20 \mathrm{ppb}$ and provide the lowest known upper limits for $\mathrm{PH}_{3}$ in the atmosphere of Venus.
\end{abstract}

Key words. planets and satellites: atmospheres - infrared: planetary systems - techniques: spectroscopic - methods: data analysis

\section{Introduction}

In light of the recent publication of Greaves et al. (2020a) reporting on the detection of phosphine above the Venusian clouds, we decided to explore the Solar Occultation at Infrared (SOIR) database in search of a spectral signature of $\mathrm{PH}_{3}$ in the infrared (IR) range. Greaves et al. (2020a) identified a phosphine line in spectra taken by two different instruments, James Clerk Maxwell Telescope (JCMT) and Atacama Large Millimetre/submillimetre Array (ALMA) in June 2017 and March 2019, respectively. They claim that it corresponds to $20 \mathrm{ppb}$ of phosphine at an altitude of 53 to $61 \mathrm{~km}$. In addition to the difficulties in understanding how phosphine can be present in Venus' atmosphere, this possible detection still needs confirmation and validation from other spectral lines of phosphine.

In this framework, Encrenaz et al. (2020) used a mid-IR $\left(950 \mathrm{~cm}^{-1}\right)$ spectrum from the Texas Echelon CrossEchelle Spectrograph (TEXES) instrument acquired on March 28, 2015. They could not detect any spectral signatures of phosphine and derived an upper limit of $5 \mathrm{ppb}$. Snellen et al. (2020) reprocessed the ALMA spectra and show that the apparent presence of a phosphine line might actually be a spurious feature from the calibration of the spectra. More recently, Villanueva et al. (2020) have concluded that the line in the JCMT spectra might be due to a $\mathrm{SO}_{2}$ line and that the calibration of ALMA spectra used in Greaves et al. (2020a) might not have been correctly performed. Using updated and corrected ALMA data, Greaves et al. (2020b) revised their conclusion, confirming the detection of $\mathrm{PH}_{3}$ but reducing the observed abundance to a $1 \mathrm{ppb}$ global disk average.

The SOIR instrument has already proven to be very sensitive to the detection and quantification of trace gases (Wilquet et al. 2012; Vandaele et al. 2015; Mahieux et al. 2015a,b) thanks to its solar occultation measurements delivering spectra with a high signal-to-noise ratio $(\mathrm{S} / \mathrm{N})$. Providing that the species have a spectral signature in the SOIR instrument's spectral range, the SOIR dataset can corroborate the possible presence of trace gases in the atmosphere of Venus or, otherwise, help to constrain their upper limits.

\section{SOIR description}

The SOIR instrument (Nevejans et al. 2006) onboard the ESA Venus Express (VEx) spacecraft (Titov et al. 2006), which is an IR spectrometer sensitive from 2.2 to $4.3 \mu \mathrm{m}$, probed the atmosphere of Venus from June 2006 until December 2014. During this time, it performed more than 750 solar occultations of Venus' middle and upper atmosphere $(\sim 60$ to $\sim 180 \mathrm{~km})$.

SOIR spectra have a resolution varying from 0.11 to $0.21 \mathrm{~cm}^{-1}$ (resolving power of 21500) with increasing wavenumber, the highest onboard VEx. SOIR combined an echelle grating to disperse the light in diffraction orders and an acousto-optical tunable filter (AOTF) as a diffraction order-sorting device. The echelle grating and the AOTF led to a division of the spectral 
Table 1. SOIR diffraction order to wavenumber range.

\begin{tabular}{lc}
\hline \hline Diffraction order & Wavenumber $\left[\mathrm{cm}^{-1}\right]$ \\
\hline 105 & $2346-2368$ \\
106 & $2369-2390$ \\
107 & $2391-2412$ \\
108 & $2413-2435$ \\
109 & $2436-2457$ \\
110 & $2458-2479$ \\
\hline
\end{tabular}

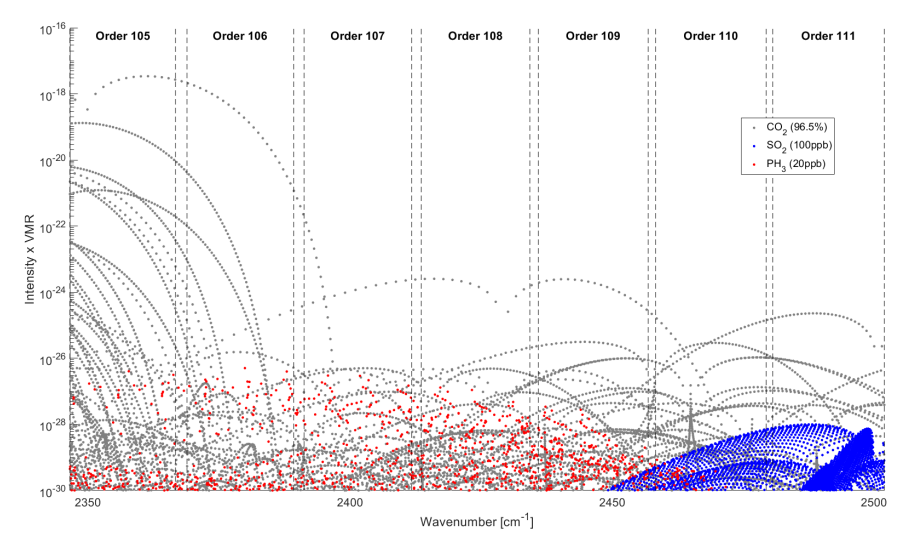

Fig. 1. Position of the $\mathrm{CO}_{2}$ (gray), $\mathrm{PH}_{3}$ (red), and $\mathrm{SO}_{2}$ (blue) rovibrational transitions in the 2348 to $2501 \mathrm{~cm}^{-1}$ region, corresponding to the SOIR 105 to 111 diffraction orders from HITRAN (Gordon et al. 2017). The line intensities were multiplied by typical Venus abundances, see legend. The SOIR orders are given at the top, with the vertical dashed lines showing their wavenumber extensions.

range ( 2256 to $4369 \mathrm{~cm}^{-1}$ ) into 94 wavenumber domains corresponding to the diffraction orders, which are simply referred to as "orders" in the following. SOIR delivered height sets of spectra per solar occultation, each of them is referred to as "dataset" hereafter (see Appendix B for more details).

We selected SOIR orders 105 to 110 (see Table 1 for the spectral range of each order) by considering the spectral signature of $\mathrm{PH}_{3}$ in the SOIR spectral range. In this region, $\mathrm{CO}_{2}$ and $\mathrm{SO}_{2}$ also have a spectral signature and Fig. 1 shows the intensities of their theoretical lines.

\section{Methods}

The following two different methods were applied to try to detect phosphine in SOIR spectra: a radiative transfer algorithm dedicated to SOIR spectra retrievals named ASIMAT and a new machine learning algorithm. A third method computed the $\mathrm{PH}_{3}$ detection limits for SOIR spectra.

ASIMAT is a Bayesian inversion algorithm using the approach developed by Rodgers (2000). It is set in an onion peeling frame, assuming a spherical symmetry of the atmosphere and fitting the logarithm of the number density of the targeted species. It accounts for the slit projected size at the impact point and for absorption line saturation (Mahieux et al. 2015a,c). ASIMAT successfully retrieved $\mathrm{CO}_{2}, \mathrm{CO}, \mathrm{HCl}, \mathrm{HF}$, $\mathrm{H}_{2} \mathrm{O}$, and $\mathrm{SO}_{2}$ from the SOIR spectra. For this study, we implemented a specific scheme to distinguish between a real detection and an upper limit value. The approach is similar to what was done in Korablev et al. (2019) for the Martian methane detection with the NOMAD-SO/ExoMars instrument. The posterior

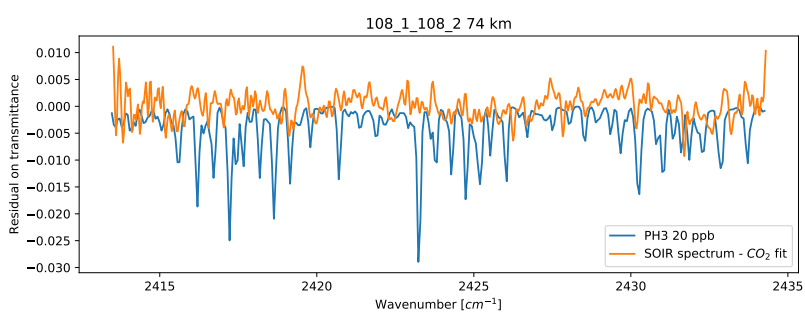

Fig. 2. Example of spectrum for orbit 108.1 order 108 bin 2 at a tangent altitude of $74 \mathrm{~km}$. The SOIR spectrum subtracted by the ASIMAT $\mathrm{CO}_{2}$ fit is plotted in orange. The synthetic spectrum of $20 \mathrm{ppb}$ of $\mathrm{PH}_{3}$ is plotted in blue.

error covariance matrix was computed as the sum of the covariance matrices of the smoothing error and the retrieval noise error (see Eq. (3.31) from Rodgers 2000). The square root of the diagonal of this matrix returned the retrieval detection limit at each altitude for a given species. We assimilated a retrieved number density lower than 3.2 times this value as a non-detection. We computed two inversions for each spectral set: the first one by considering $\mathrm{CO}_{2}$ and $\mathrm{PH}_{3}$ (and $\mathrm{SO}_{2}$ for order 110), and the second one by considering only $\mathrm{CO}_{2}$. We compared the root mean square (rms) for each spectrum fit, and only kept the ones that have a lower rms when retrieving $\mathrm{CO}_{2}+\mathrm{PH}_{3}+\mathrm{SO}_{2}$ than when only retrieving $\mathrm{CO}_{2}+\mathrm{SO}_{2}$.

An alternative attempt to detect $\mathrm{PH}_{3}$ was carried out with a machine learning tool that had already been used to detect the $\mathrm{CO}_{2}$ quadrupole and to infer the absence of methane in NOMAD-SO spectra (Schmidt et al. 2020). The method aims to summarize the dataset with statistical endmembers, hereafter "sources". First, data are pretreated to remove the baseline and converted into absorbance. The method consists of a linear blind source separation under positivity constraints and uses the probabilistic sparse Non-negative Matrix Factorization (psNMF) described in Hinrich \& Mørup (2018). Appendix E provides more information on this analysis.

In addition to these detection methods, we determined the detection limits (DLs) of $\mathrm{PH}_{3}$ in SOIR spectra in the volume mixing ratio (VMR). For each SOIR spectrum, we compared the measurement noise to a synthetic spectrum of $\mathrm{PH}_{3}$ simulated by considering the SOIR instrumental function. We considered that a clear detection of a $\mathrm{PH}_{3}$ line in SOIR spectra should be at least 3.2 times higher than the measurement noise. Appendix F contains a description of this method.

\section{Results}

Neither the radiative transfer algorithm nor the machine learning algorithm could infer any real detection of $\mathrm{PH}_{3}$ lines for any of the orders selected. In orange, Fig. 2 shows an example of the residual of the SOIR spectrum at $74 \mathrm{~km}$ subtracted by the ASIMAT $\mathrm{CO}_{2}$ fit for order 108 . We see that several lines of the synthetic $\mathrm{PH}_{3}$ spectrum (blue) are already much stronger than the residual. We see no clear presence of $\mathrm{PH}_{3}$ lines in the SOIR spectrum.

Figure 3 shows an example from the machine learning algorithm for order 106 where one of the sources is clearly identified as $\mathrm{CO}_{2}$ (above panel), but no source could be identified as $\mathrm{PH}_{3}$. In the lower panel, the blue curve corresponds to a source with the highest contribution $A_{\mathrm{PH}_{3}}$ to a synthetic $\mathrm{PH}_{3}$ spectrum (black curve). 

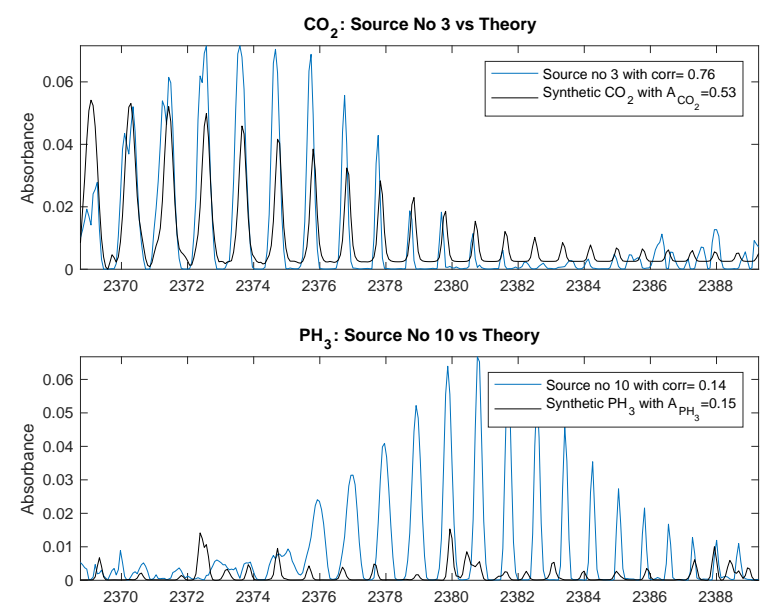

Fig. 3. Example of source derived from the machine learning algorithm (in blue) compared to synthetic spectrum (in black) for order 106. The synthetic spectrum is rescaled with fitted linear contribution of $\mathrm{CO}_{2}$ $\left(A_{\mathrm{CO}_{2}}\right)$ and $\mathrm{PH}_{3}\left(A_{\mathrm{PH}_{3}}\right)$. The correlation coefficient between source and synthetic spectra is noted corr. Above, the source is identified as $\mathrm{CO}_{2}$ (significant $A_{\mathrm{CO}_{2}}>0.5$ and high corr $>0.3$ ). Below the closest source (highest $A_{\mathrm{PH}_{3}}$ ) to the $\mathrm{PH}_{3}$ synthetic spectrum is not coherent with its presence $\left(A_{\mathrm{PH}_{3}}<0.5\right.$ and corr $\left.<0.3\right)$.

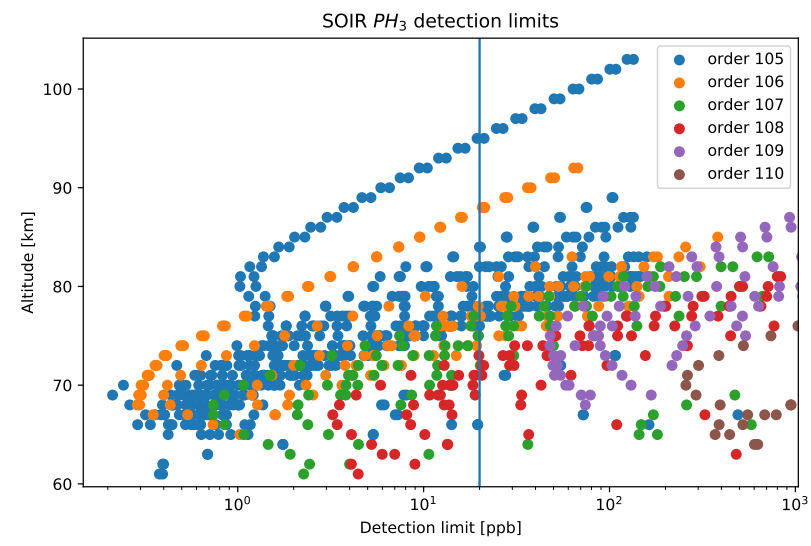

Fig. 4. Detection limits as a function of altitude. Colors are the different diffraction orders of SOIR. The blue vertical line corresponds to $20 \mathrm{ppb}$. The spectral range of the diffraction orders are provided in Table 1.

The DLs were computed for two cases. The first case is a constant $\mathrm{PH}_{3}$ VMR up to $190 \mathrm{~km}$. The second case is a constant $\mathrm{PH}_{3} \mathrm{VMR}$ up to $68 \mathrm{~km}$. The choice of this last case comes from the top panel of Fig. 9 from Greaves et al. (2020a) where $68 \mathrm{~km}$ corresponds to the highest altitude where the $\mathrm{PH}_{3} \mathrm{VMR}$ is higher than $0.1 \mathrm{ppb}$.

Figure 4 shows the DL profiles for orders 105 to 110 for the first case. They have the typical shape expected for solar occultation measurements: from higher altitudes, they decrease with lower tangent altitudes until they increase again because of the progressive presence of clouds and hazes, which decreases the $\mathrm{S} / \mathrm{N}$ along the whole spectra. The tangent altitude of lowest detection limit (ALDL) varies from one occultation to another depending on the latitude covered, the atmosphere variability, the cloud-deck height, and the strength of the $\mathrm{PH}_{3}$ lines in the spectral order sounded.

The DLs for orders 109 and 110 are above $20 \mathrm{ppb}$, but those for orders 105 to 108 are well below $20 \mathrm{ppb}$. We expect some strong variations in the DLs with respect to the diffraction orders.

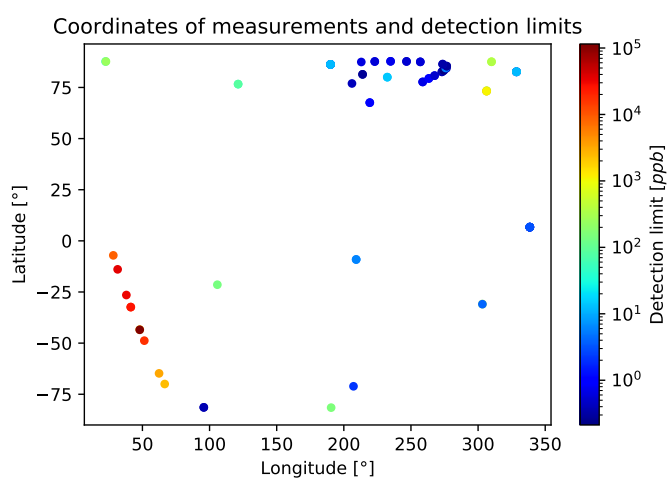

Fig. 5. Latitude and longitude coverage of ALDL for SOIR datasets.

The $\mathrm{PH}_{3}$ line intensities are stronger in orders 105 and 106 and decrease in orders 107 to 110 . Figure 4 clearly shows this variation in $\mathrm{PH}_{3}$ lines intensities.

The altitude of interest is closer to $60 \mathrm{~km}$ and thus a few kilometers below the typical ALDL. For order 108, the lowest DL is $3 \mathrm{ppb}$ at $67 \mathrm{~km}$ and $4 \mathrm{ppb}$ at $61 \mathrm{~km}$ (the lowest tangent altitude probed). For order 107, the lowest DL is $0.7 \mathrm{ppb}$ at $67 \mathrm{~km}$ and $2 \mathrm{ppb}$ at $61 \mathrm{~km}$. For order 106, the lowest DL is $0.3 \mathrm{ppb}$ at $69 \mathrm{~km}$ and $1 \mathrm{ppb}$ at $65 \mathrm{~km}$. For order 105, the lowest DL is $0.2 \mathrm{ppb}$ at $69 \mathrm{~km}$ and $0.4 \mathrm{ppb}$ at $61 \mathrm{~km}$.

The DLs lower than $20 \mathrm{ppb}$ and below $62 \mathrm{~km}$ corresponds to ten different datasets of SOIR and five different occultations. The DLs below $20 \mathrm{ppb}$ and below $61 \mathrm{~km}$ correspond to six datasets from four different occultations.

The SOIR datasets presented here mostly cover latitudes above $60^{\circ}$ north. Still eleven datasets cover the latitudes below $60^{\circ}$, corresponding to three different occultations. As seen in Fig. 5, they correspond to latitudes $6.67^{\circ}$ (orbit 108.1, orders 105 to 108 ),$-30.96^{\circ}$ (orbit 446.1 , order 108 ), and $-9.11^{\circ}$ (orbit 591.1 , order 105). For the dataset corresponding to orbit 446.1 order 108 bin 2, the ALDL is at $62 \mathrm{~km}$ with a detection limit of $8 \mathrm{ppb}$ and at $61 \mathrm{~km}$, and the DL is still $9 \mathrm{ppb}$. For orbit 108.1 order 107 bin 2, the DL at $62 \mathrm{~km}$ is $7 \mathrm{ppb}$.

ASIMAT also derives DLs and the results are the same as the ones shown in this section. The machine learning algorithm DL is estimated from those derived in Schmidt et al. (2020) for $\mathrm{CH}_{4}$ lines at $3067.3 \mathrm{~cm}^{-1}$, which are lost in dominant $\mathrm{H}_{2} \mathrm{O}$ lines in a realistic synthetic dataset, including nonlinear radiative transfer and an instrumental effect. In this case, the detection limit was with a noise variance of 0.001 between 500 ppt (mean absorption bands $=0.0092, S / N=0.9$ ) and $100 \mathrm{ppt}$ (mean absorption bands $=0.00018, S / N=0.1$ ). Assuming the same behavior, we estimate that the machine learning algorithm $\mathrm{PH}_{3}$ DL for SOIR spectra is thus $0.5 \mathrm{ppb}$. This value is similar to the lowest DL in Table 2.

For the second case (no $\mathrm{PH}_{3}$ above $68 \mathrm{~km}$ ), there are two orbits with DLs below 20 ppb: orbit 108.1 and 1250.1. Table 2 summarizes the DLs for a VMR of phosphine until $68 \mathrm{~km}$ (column DL2). For comparison, the corresponding DLs of the first case are also provided in column DL1.

\section{Discussion and conclusions}

We did not detect any phosphine in the SOIR spectra using the two completely different and independent detection methods described above. By considering a constant phosphine VMR, we derived SOIR DLs as low as $0.2 \mathrm{ppb}$ at $69 \mathrm{~km}$ with still multiple DLs lower than 20 ppb from 60 to $95 \mathrm{~km}$ (see Fig. 4). For a more 
Table 2. SOIR detection limits below $20 \mathrm{ppb}$ for a constant VMR of phosphine below $180 \mathrm{~km}$ (DL1) and $68 \mathrm{~km}$ (DL2) and no phosphine above the respective altitude.

\begin{tabular}{lccc}
\hline \hline Orbit.case-order(bin) & $\begin{array}{c}\text { Altitude } \\
{[\mathrm{km}]}\end{array}$ & $\begin{array}{c}\text { DL1 } \\
{[\mathrm{ppb}]}\end{array}$ & $\begin{array}{c}\text { DL2 } \\
{[\mathrm{ppb}]}\end{array}$ \\
\hline $108.1-106(2)$ & 65 & 1 & 2 \\
\hline $108.1-107(1)$ & 64 & 4 & 6 \\
\hline \multirow{2}{*}{$108.1-107(2)$} & 62 & 2 & 3 \\
& 63 & 2 & 3 \\
\hline $108.1-108(1)$ & 64 & 1 & 3 \\
\hline \multirow{2}{*}{$108.1-108(2)$} & 64 & 13 & 18 \\
\hline $1250.1-105(1)$ & 62 & 9 & 10 \\
\hline $1250.1-105(2)$ & 63 & 7 & 9 \\
\hline
\end{tabular}

stringent case of no phosphine above $68 \mathrm{~km}$, a constant volume mixing ratio below $68 \mathrm{~km}$, and by considering only the latitudes between $-60^{\circ}$ and $60^{\circ}$, the lowest DL is $2 \mathrm{ppb}$ at $65 \mathrm{~km}$.

There is a difference in the time of measurements as SOIR datasets with DLs lower than 20 ppb extend from August 2006 until January 2010 and the spectra used by Greaves et al. (2020a) were recorded in June 2017 and March 2019. Another difference is that SOIR scanned a localized region of the Venus terminator, while the spectra from Greaves et al. (2020a) cover more than $15^{\circ}$ of latitude in the Venus disk, as seen from the Earth. These differences might be important if phosphine is really present in Venus' atmosphere and if the process producing phosphine is localized and varies in time.

Acknowledgements. Venus Express is a planetary mission from the European Space Agency (ESA). We wish to thank all ESA members who participated in the mission, in particular, H. Svedhem and D. Titov. The research program was supported by the Belgian Federal Science Policy Office and the European Space Agency (ESA, PRODEX program, contracts C 90268, 90113, 17645, 90323, and 4000107727). A. Mahieux was supported by the Marie Sklodowska-Curie Action from the European Commission under grant number 838587. This work was supported by the Belgian Fonds de la Recherche Scientifique - FNRS under grant number 30442502 (ET-HOME). SR thanks BELSPO for the FED-tWIN funding (Prf-2019-077 - RT-MOLEXO). F. Schmidt acknowledges support from the "Institut National des Sciences de 1'Univers" (INSU), the "Centre National de la Recherche Scientifique" (CNRS) and "Centre National d'Etudes Spatiales" (CNES) through the "Programme National de Planétologie". We wish to thank the referee for his valuable comments and suggestions that improved the readability of this Letter.

\section{References}

Butler, R. A., Sagui, L., Kleiner, I., \& Brown, L. R. 2006, J. Mol. Spectr., 238 , 178

Chamberlain, S., Mahieux, A., Robert, S., et al. 2020, Icarus, 346, 113819

Encrenaz, T., Greathouse, T., Marcq, E., et al. 2020, A\&A, 643, L5

Gordon, I. E., Rothman, L. S., Hill, C., et al. 2017, J. Quant. Spectr. Rad. Transf., 203, 3

Greaves, J. S., Richards, A. M., Bains, W., et al. 2020a, Nat. Astron., in press [arXiv:2009.06593]

Greaves, J. S., Richards, A. M. S., Bains, W., et al. 2020b, ArXiv e-prints [arXiv:2011.08176]

Hinrich, J. L., \& Mørup, M. 2018, in Probabilistic Sparse Non-negative Matrix Factorization (Springer Verlag), Lect. Notes Comput. Sci., 10891, 488

IUPAC 2008, IUPAC Compendium of Chemical Terminology (IUPAC)

Keating, G. M., Bertaux, J. L., Bougher, S. W., et al. 1985, Adv. Space Res., 5, 117

Korablev, O., Vandaele, A. C. A. A. C., Montmessin, F., et al. 2019, Nature, 568, 517

Mahieux, A., Vandaele, A., Bougher, S., et al. 2015a, Planet. Space Sci., 113,309

Mahieux, A., Wilquet, V., Vandaele, A. C., et al. 2015b, Planet. Space Sci., 113,264

Mahieux, A., Vandaele, A. C., Robert, S., et al. 2015c, Planet. Space Sci., 113,347

Mahieux, A., Vandaele, A. C., Robert, S., et al. 2015d, Planet. Space Sci., 113,193

Nevejans, D., Neefs, E., Van Ransbeeck, E., et al. 2006, Appl. Opt., 45, 5191

Nikitin, A. V., Ivanova, Y. A., Rey, M., et al. 2017, J. Quant. Spectr. Rad. Transf., 203, 472

Rodgers, C. D. 2000, in Inverse Methods for Atmospheric Sounding (World Scientific), Ser. Atmos. Ocean. Planet. Phys., 2, 238

Schmidt, F., Mermy, G. C., Erwin, J., et al. 2020, J. Quant. Spectr. Rad. Transf., 259, 107361

Snellen, I. A. G., Guzman-Ramirez, L., Hogerheijde, M. R., Hygate, A. P. S., \& van der Tak, F. F. S. 2020, A\&A, 644, L2

Tarrago, G., Lacome, N., Lévy, A., et al. 1992, J. Mol. Spectr., 154, 30

Titov, D. V., Svedhem, H., McCoy, D., et al. 2006, Cosm. Res., 44, 334

Trompet, L., Mahieux, A., Ristic, B., et al. 2016, Appl. Opt., 55, 9275

Vandaele, A. C., Mahieux, A., Robert, S., et al. 2013, Opt. Exp., 21, 21148

Vandaele, A. C., Neefs, E., Drummond, R., et al. 2015, Planet. Space Sci., 119, 233

Villanueva, G., Cordiner, M., Irwin, P., et al. 2020, Nat. Astron., submitted [arXiv:2010.14305]

Wilquet, V., Drummond, R., Mahieux, A., et al. 2012, Icarus, 217, 875

Zasova, L. V., Ignatiev, N., Khatuntsev, I., \& Linkin, V. 2007, Planet. Space Sci., 55, 1712 


\section{Appendix A: The SOIR instrument}

The SOIR detector counted 320 columns of pixels along its wavenumber axis and 24 illuminated rows (among 256 in total) along its spatial axis. The spectral width of a pixel varied from 0.06 to $0.12 \mathrm{~cm}^{-1}$ with increasing order. The instrument slit was aligned to the Venus limb, such that the detector's spatial axis was parallel to the limb. Because of telemetry limitations, all of the illuminated rows along the spatial axis were not transmitted to Earth, but they were summed up on board in two spatial bins, thus taken at two slightly different altitudes. The computed wavenumber interval associated with the detector pixels 0 and 319 for orders 101 to 194 can be found in Vandaele et al. (2013).

The AOTF transfer function full-width at half-maximum (FWHM) was larger than the order free spectral range $\left(\sim 24 \mathrm{~cm}^{-1}\right)$, which induced leaking of the signal from adjacent orders in the targeted order, modulated by the AOTF transfer function shape. The AOTF transfer function had the approximate shape of a sinc-squared function and a FWHM of $\sim 24 \mathrm{~cm}^{-1}$. This effect slightly reduces the instrument sensitivity for targeted orders that are close to bands stronger by several orders of magnitude.

The instantaneous field of view, corresponding to the vertical extent of the projected slit at the Venus limb during a single measurement, varied with the latitude of the observation due to the elliptical shape of the VEx orbit around Venus. It ranged from $200 \mathrm{~m}$ for northern polar observations to $5 \mathrm{~km}$ at the South Pole. The vertical sampling, that is to say the vertical distance between two successive measurements of the same bin of the same order, was also latitude dependent: it varied from $2 \mathrm{~km}$ at the North Pole, $500 \mathrm{~m}$ between $40^{\circ}$ and $70^{\circ}$ north, and up to $5 \mathrm{~km}$ at the South Pole.

\section{Appendix B: SOIR datasets}

The transmittance spectra analyzed in this study were obtained through an improved algorithm described in Trompet et al. (2016). The transmittances and related uncertainties were calculated by dividing the signal in the penumbra region by an extrapolated reference calculated from a linear regression in the full Sun region (Vandaele et al. 2013).

During a solar occultation, since the light beam probed deeper layers in the atmosphere, the following two processes reducing the transmittance took place: extinction by aerosols and absorption by trace gases. Each occultation probed four different wavelength regions, allowing us to examine several atmospheric constituents simultaneously. Therefore, the SOIR dataset contains eight independent sets of spectra, corresponding to the four orders and two spatial bins. By combining the profiles retrieved from each dataset, high-resolution vertical profiles of the concentration of various elements were retrieved. Moreover, since SOIR was sensitive to $\mathrm{CO}_{2}$, the Venus major constituent, we could compute the temperature profile for each occultation assuming hydrostatic equilibrium.

Several papers have been devoted to main molecules that have a spectral signature in the SOIR spectral range and present in Venus' atmosphere, such as $\mathrm{H}_{2} \mathrm{O}$ (Chamberlain et al. 2020), $\mathrm{CO}_{2}$ (Mahieux et al. 2015a), CO (Vandaele et al. 2015), $\mathrm{SO}_{2}$ (Mahieux et al. 2015a), hydrogen halides $(\mathrm{HCl}, \mathrm{HF}$, see Mahieux et al. 2015d), and the aerosols (Wilquet et al. 2012).

Table B. 1 lists the orbits that targeted orders 105 to 110 . The SOIR detector lines in the spatial direction are summed up in two spectral bins, simply called bins. An orbit and a case number reference each occultation. The case number corresponds
Table B.1. Set of SOIR observations used in this analysis.

\begin{tabular}{|c|c|c|c|c|}
\hline Date & Orbit.case & Orders & $\begin{array}{c}\text { Latitude } \\
{\left[^{\circ}\right]}\end{array}$ & $\begin{array}{c}\text { Longitude } \\
{\left[{ }^{\circ}\right]}\end{array}$ \\
\hline $27 / 05 / 2006$ & 36.1 & $109-110$ & 73 & 306 \\
\hline 07/08/2006 & 108.1 & $105-108$ & 7 & 339 \\
\hline 28/08/2006 & 129.1 & $105-108$ & 86 & 190 \\
\hline 04/09/2006 & 136.1 & 106 & 80 & 233 \\
\hline $02 / 12 / 2006$ & 225.1 & $105-108$ & 83 & 329 \\
\hline $12 / 12 / 2006$ & 235.1 & 110 & 88 & 310 \\
\hline $19 / 12 / 2006$ & 242.1 & 107 & -71 & 207 \\
\hline $11 / 07 / 2007$ & 446.1 & 108 & -31 & 303 \\
\hline $23 / 07 / 2007$ & 458.1 & 108 & 87 & 293 \\
\hline $03 / 12 / 2007$ & 591.1 & 105 & -9 & 209 \\
\hline $17 / 02 / 2008$ & 667.1 & 105 & 78 & 259 \\
\hline $19 / 02 / 2008$ & 669.1 & 105 & 79 & 263 \\
\hline $21 / 02 / 2008$ & 671.1 & 105 & 81 & 268 \\
\hline $24 / 02 / 2008$ & 674.1 & 105 & 83 & 273 \\
\hline $25 / 02 / 2008$ & 675.1 & 105 & 83 & 275 \\
\hline $27 / 02 / 2008$ & 677.1 & 105 & 84 & 277 \\
\hline $29 / 02 / 2008$ & 679.1 & 105 & 85 & 277 \\
\hline $02 / 03 / 2008$ & 681.1 & 105 & 86 & 274 \\
\hline $05 / 03 / 2008$ & 684.1 & 105 & 88 & 257 \\
\hline 06/03/2008 & 685.1 & 105 & 88 & 247 \\
\hline $07 / 02 / 2008$ & 686.1 & 105 & 88 & 235 \\
\hline $08 / 02 / 2008$ & 687.1 & 105 & 88 & 223 \\
\hline $09 / 02 / 2008$ & 688.1 & 105 & 87 & 213 \\
\hline $24 / 03 / 2008$ & 703.1 & 105 & 77 & 206 \\
\hline $30 / 03 / 2008$ & 709.1 & 105 & 67 & 219 \\
\hline $16 / 05 / 2009$ & 1121.1 & 106 & 81 & 214 \\
\hline 27/08/2009 & 1224.1 & 110 & 28 & 180 \\
\hline $27 / 08 / 2009$ & 1224.2 & 110 & 70 & 181 \\
\hline 22/09/2009 & 1250.1 & 105 & -81 & 96 \\
\hline $23 / 09 / 2009$ & 1251.1 & 109 & 88 & 23 \\
\hline $25 / 01 / 2010$ & 1375.1 & $107-108$ & -21 & 106 \\
\hline 26/01/2010 & 1376.1 & 109 & 76 & 121 \\
\hline $01 / 09 / 2013$ & 2690.1 & 110 & 60 & 160 \\
\hline $01 / 09 / 2013$ & 2690.2 & 110 & 9 & 158 \\
\hline $03 / 09 / 2013$ & 2692.1 & 110 & -7 & 152 \\
\hline $04 / 09 / 2013$ & 2693.1 & 110 & -14 & 148 \\
\hline $06 / 09 / 2013$ & 2695.1 & 110 & -26 & 142 \\
\hline 07/09/2013 & 2696.1 & 110 & -32 & 139 \\
\hline $09 / 09 / 2013$ & 2698.1 & 110 & -43 & 132 \\
\hline $10 / 09 / 2013$ & 2699.1 & 110 & -49 & 129 \\
\hline $13 / 09 / 2013$ & 2702.1 & 110 & -65 & 118 \\
\hline $14 / 09 / 2013$ & 2703.1 & 110 & -70 & 113 \\
\hline
\end{tabular}

Notes. The first column gives the orbit number and case, the second lists the orders sensitive to $\mathrm{PH}_{3}$, and the last two columns give the latitude and east longitude. The orbit number is followed with a case number specifying the different measurements along the same orbit of VEx around Venus.

to the measurement sequence along the orbit. Thus, during a solar occultation, SOIR scanned four diffraction orders and recorded two sets of spectra (referenced by a "bin" number) per diffraction order. Therefore, SOIR delivered height sets of spectra per occultation, each of them is referred to as "dataset". Each SOIR dataset is referenced by four numbers: an orbit number, a case number, a diffraction order, and a bin number. For instance, we write orbit 446 case 1 order 108 bin 2 as 446.1-108(2). 


\section{Appendix C: $\mathrm{PH}_{3}$ absorption lines in SOIR spectral range}

Phosphine is a trigonal pyramidal molecule with $\mathrm{C}_{3 v}$ molecular symmetry. The molecule has four fundamental normal modes of vibration at $2321 \mathrm{~cm}^{-1}, 992 \mathrm{~cm}^{-1}, 2327 \mathrm{~cm}^{-1}$, and $1118 \mathrm{~cm}^{-1}$. The IR bands of $\mathrm{PH}_{3}$ were measured in the laboratory between 4 and $5 \mu \mathrm{m}$ by Tarrago et al. (1992) and by Butler et al. (2006) between 2.8 and $3.7 \mu \mathrm{m}$. The HITRAN online $\mathrm{PH}_{3}$ line list was significantly updated in October 2020 and now encompasses the spectral regions from 0 to $3700 \mathrm{~cm}^{-1}$ (see hitran.org for the last update of $\mathrm{PH}_{3}$ lines). These new values originate from ab initio calculations performed by the TheoReTS team (M. Rey, priv. comm.) and by calculations using an effective Hamiltonian approach by Nikitin et al. (2017).

\section{Appendix D: Radiative transfer algorithm ASIMAT}

ASIMAT retrieves the logarithm of the species density from each of the eight spectral sets independently. It assumes a covariance of 0.25 . The Jacobians are derived analytically. The continuous background, which is dependent on the atmospheric aerosol loading, is modeled using a fifth-order polynomial. The spectral line information was taken from HITRAN (Gordon et al. 2017) and precomputed by a ro-vibrational band on a temperature and pressure grid. We used the $\mathrm{CO}_{2}$ density profile from the VAST compilation (Mahieux et al. 2015a) as an a priori profile. For $\mathrm{SO}_{2}$ and $\mathrm{PH}_{3}$, we used a constant volume mixing ratio profile, which is equal to $70 \mathrm{ppb}$ for $\mathrm{SO}_{2}$ and $10 \mathrm{ppb}$ for $\mathrm{PH}_{3}$. The final profiles correspond to a combination of the derived profile from each individual dataset (for an order and spatial bin), which were combined using an error weighted moving average function. ASIMAT also returns an upper-limit vertical profile based on the retrieval noise (see Mahieux et al. 2015d for a full description). Using ASIMAT, we focused on order 107 to 110 because the strong $\mathrm{CO}_{2}$ bands from orders 105 and 106 are more arduous to fit for the low altitudes targeted in this study.

\section{Appendix E: Machine learning detection tool psNMF}

This algorithm has already been described in Schmidt et al. (2020). For each diffraction order, the whole set of spectra is analyzed at once by considering that the spectra are a combination of three, four, five, or ten endmembers (or also called "sources"). If the chemical species are well mixed, one single source with the average mixture should appear. If the compounds are not well mixed, that is to say if there are significant statistical variations in abundances, each pure compound should appear in the sources. Due to nonlinearity, sources are often duplicated with a very similar shape. Since the spectra signature of minor species and its variability is relatively small, we tested the analysis for up to ten sources, even only two or three are expected $\left(\mathrm{CO}_{2}, \mathrm{PH}_{3}\right.$, and $\mathrm{SO}_{2}$ for order 109 and 110).

For the not well mixed scenario, a potential detection occurs if one of the source shows a significant correlation higher than 0.3 with a pure $\mathrm{PH}_{3}$ spectrum. For the well mixed scenario, we also computed the linear contribution of the pure spectra $P$ for each source $S$ by estimating $A$, following this equation:

$S=A_{\mathrm{CO}_{2}} P_{\mathrm{CO}_{2}}+A_{\mathrm{PH}_{3}} P_{\mathrm{PH}_{3}}+A_{\mathrm{SO}_{2}} P_{\mathrm{SO}_{2}}$

with $A>0$. If the source $S$ with the highest $A_{\mathrm{PH}_{3}}$ (the closest source) has a clear contribution of $\mathrm{PH}_{3}$ (presence of the major bands of $\mathrm{PH}_{3}$ ), we can infer the detection. From the analysis of the complete dataset for all orders 105 to 110 , none of the above cases were reached.

\section{Appendix F: Detection limit computation}

In this method, the volume mixing ratio of $\mathrm{PH}_{3} v m r_{\mathrm{PH}_{3}}$ is supposed to be constant with the altitude. The optical depth $\tau_{\mathrm{PH}_{3}}$ was then computed as

$$
\begin{aligned}
\tau_{\mathrm{PH}_{3}}\left(v, z_{\mathrm{tg}}\right) & =\int_{z_{\mathrm{tg}}}^{z_{\mathrm{top}}} \sigma(v, p(z), t(z)) n_{\mathrm{PH}_{3}}(z) \mathrm{d} z \\
& =v m r_{\mathrm{PH}_{3}} \int_{z_{\mathrm{tg}}}^{z_{\mathrm{top}}} \sigma(v, p(z), t(z)) n_{\mathrm{tot}}(z) \mathrm{d} z
\end{aligned}
$$

where $n_{\mathrm{PH}_{3}}$ is the $\mathrm{PH}_{3}$ number density profile, $n_{\text {tot }}$ is the total density profile by considering all species present in the atmosphere of Venus, and $\sigma$ represents the absorption coefficients depending on the pressure $p$ and the temperature $t$. Both parameters were derived from Zasova et al. (2007) for the middle atmosphere and Keating et al. (1985) for the high atmosphere with a fitting function joining them between 90 and $110 \mathrm{~km}$. We integrated along the line of sight and on all the layers lying above the tangent altitude of the measurement $\left(z_{\mathrm{tg}}\right)$. We then convolved $\tau_{\mathrm{PH}_{3}}$ with the instrument resolution. In our first derivation of DLs, we used a $z_{\text {top }}$ equal to $190 \mathrm{~km}$ and in our second derivation of the DLs, we limited $z_{\text {top }}$ to $68 \mathrm{~km}$.

The optical depth $\tau_{\mathrm{PH}_{3}}$ must be at least as important as the optical depth computed inverting the uncertainties $Y_{\text {Error }}$ on the transmittances:

$\tau_{\text {err }}\left(v, z_{\mathrm{tg}}\right)=-\ln \left(1-3.2 \frac{Y_{\text {Error }}\left(v, z_{\mathrm{tg}}\right)}{Y_{\mathrm{bg}}\left(v, z_{\mathrm{tg}}\right)} \frac{I_{\mathrm{tot}}(v)}{I_{\mathrm{o}}(v)}\right)$

where $Y_{\text {bg }}$ is the background, and $I_{\text {tot }}$ and $I_{\mathrm{o}}$ are the contributions from the central order radiance and the total radiance obtained when probing the $z_{\mathrm{tg}}$ altitude. The factor 3.2 means that the strongest line of $\mathrm{PH}_{3}$ should be at least 3.2 times higher than the noise level in order to be considered as a clear detection. Indeed, IUPAC advices providing detection limits by the mean level of detection plus 3.2 times the standard deviation on the level of detection (IUPAC 2008). We could not detect $\mathrm{PH}_{3}$, but we still multiplied the uncertainties on SOIR transmittance by 3.2 in the formula for $\tau_{\text {err }}$ to consider proper detection limits and not a limit of blank.

By considering the detection limit as the equality between $\tau_{\mathrm{PH}_{3}}$ and $\tau_{\mathrm{err}}$, we can write

$v m r_{\mathrm{PH}_{3}}\left(z_{\mathrm{tg}}\right)=\min _{v} \frac{\tau_{\mathrm{err}}}{\int_{z_{\mathrm{tg}}}^{z_{\mathrm{top}}} \sigma(v, p(z), t(z)) n_{\mathrm{tot}}(z) \mathrm{d} z}$.

We can then build a vertical profile of detection limits because each transmittance spectrum corresponds to a different tangent altitude. 